\title{
In vitro measurement of nuclear permeability changes in apoptosis
}

\author{
Sebastian Roehrig, ${ }^{1}$ Anja Tabbert, ${ }^{1}$ and Elisa Ferrando-May* \\ Molecular Toxicology Group, Department of Biology, University of Konstanz, P.O. Box X911, 78457 Konstanz, Germany
}

\begin{abstract}
In the eukaryotic cell, exchange of biomolecules between nucleus and cytoplasm is a highly regulated process which responds sensitively to changes of the environment. One well known cellular response to environmental challenges is cell death by apoptosis. In fact, apoptosis has been shown to affect the nucleocytoplasmic transport machinery, in particular the nuclear pore, by modulating its size exclusion limit for passive diffusion. The underlying molecular factors are still unknown, mainly because of the lack of a suitable system to detect and quantitate the apoptotic effects on the nuclear pore. Here we present an assay that was designed to measure alterations of the permeability of the nuclear envelope under apoptotic conditions. The assay is based on the well estab lished technique of selective permeabilization of the plasma membrane with digitonin and allows assessment of permeability changes in nonfixed samples. It comprises a computer program, called Nuclear Permeability Assay, for the quantitation of the nuclear fluorescence signal, which may be generally employed for the evaluation of in vitro transport systems using semipermeabilized cells, such as assays for nuclear import and export.
\end{abstract}

Keywords: Nuclear pore; Nuclear transport; Dextran; Digitonin; Confocal microscopy; Fluorescence imaging

The nuclear envelope is a double-membrane barrier which controls the movement of ions, small solutes, and macromolecules between the nucleus and the cytoplasm. Passage through the nuclear envelope occurs via nuclear pore complexes, huge macromolecular structures spanning both membranes of the envelope. They consist of a proteinaceous framework with eightfold rotational symmetry enclosing an aqueous transport channel of about $510 \mathrm{~nm}$ in diameter. Small molecules below 20 $40 \mathrm{kDa}$ can freely diffuse through this channel while larger species are transported via an active, signalmediated process [l 13$]$.

Exchange of material by both passive and active translocation is essential to the eukaryotic cell. Thus, it is not surprising that this function is tightly coupled to the physiological and metabolic status of the cell. Indeed there is accumulating evidence that both the physical diameter of the nuclear pore, determining the size exclusion limit for passive diffusion, and the capacity for active translocation

\footnotetext{
${ }^{*}$ Corresponding author. Fax: +49 7531884033.

Email address: Elisa.May@uni konstanz.de (E. Ferrando May).

${ }^{1}$ These authors contributed equally to this work.
}

can vary with overall cellular activity. Early studies suggested that growth factor stimulation of untransformed murine fibroblasts augments passive nuclear permeability [4], while fluctuations in the size of the passive diffusion channel were observed during the cell cycle of HeLa cells [5]. The functional diameter for signal-mediated nuclear uptake was shown to decrease by $58 \mathrm{~nm}$ when a cell transits from a proliferating to a quiescent state. This effect may be reverted by inducing cellular differentiation or by transformation with viral oncogenes [5 7]. It was proposed that under conditions of low metabolic activity, the cell might restrict the capacity of the nuclear pore to dilate during the process of active translocation, thereby downregulating nucleocytoplasmic trafficking. In fact, during signal-mediated transport the nuclear pore has been demonstrated to undergo impressive structural rearrangements which allow for the accommodation of particles of several million Dalton [8 10$]$.

One factor that has been shown to influence the conformation of the nuclear pore is the level of calcium in the nuclear envelope. Depletion of nuclear envelope calcium stores causes morphological changes in the central channel of the nuclear pore as observed by 
atomic force microscopy [11 13]. Moreover, it blocks passive diffusion of intermediate-sized dextrans [14,15]. Whether depletion of intracellular calcium stores also affects signal-mediated transport in intact cells remains controversial $[16,17]$.

Cell shape, too, has been suggested to influence nuclear permeability and transport capacity. Accordingly, nuclear pores in rounded cells were found to have a smaller functional diameter and decreased passive permeability compared to pores of flattened cells $[4,18]$.

Among the physiological responses of the cell which may impinge on nucleocytoplasmic transport are not only proliferation and differentiation but also cell death by apoptosis. In many cellular systems, apoptosis is not only associated with an overall downregulation of gene expression activity [19], but also accompanied by alterations of the cytoskeleton [20] and changes in intracellular calcium concentration [21]. Thus it seems likely that nuclear transport capacity may be modulated as part of the apoptotic cell death program. In fact, we have previously shown that the localization of crucial components of the nuclear transport machinery, such as Ran and the importin receptors $\alpha$ and $\beta$, is deregulated at the onset of apoptosis. This is paralleled by an increase in the size exclusion limit for passive permeability of the nuclear envelope, as measured by microinjection of fluorescent $70 \mathrm{kDa}$ dextran into living cells [22]. Similar results were also described for cells expressing a green fluorescent protein dimer as a probe for passive diffusion [23].

In this paper we report on a novel method to quantitate alterations of the passive permeability of the nucleus during apoptosis. The method is based upon the in vitro assay established by Adam et al. [24] for the analysis of nuclear protein import. It relies upon the selective permeabilization of the plasma membrane at low concentrations of the detergent digitonin, which leaves the nuclear membrane intact. In these semipermeabilized cells the properties of the nuclear envelope, including the permeability for fluorescently labeled reporter molecules, can be investigated directly, without the need for microinjection or cell transfection. The method includes a custom-designed computer software for the semiautomatic evaluation of nuclear fluorescence intensity. As shown here, this method allows one to reproduce and quantitate the effects previously observed in intact cells and provides a tool for identifying the molecular factors that underlie the modulation of passive nuclear permeability during apoptosis.

\section{Materials and methods}

\section{Materials}

Digitonin was obtained from Sigma at the highest available purity and dissolved in water at $20 \mathrm{mg} / \mathrm{ml} ; 70-$
$\mathrm{kDa}$ Texas red-labeled dextran was from Molecular Probes. CD95 ligand was obtained as culture supernatant of Neuro-2a cells transfected with murine CD95 ligand cDNA [25].

\section{Cell culture and isolation of apoptotic extracts}

Jurkat human T cells (clone E6-1) were grown in RPMI-1640, and HeLa 229 cells in Dulbecco's modified Eagle's medium, both media supplemented with $10 \%$ fetal calf serum, at $37^{\circ} \mathrm{C}$ in a humidified atmosphere. For induction of apoptosis Jurkat cells were incubated in serum-free medium at a density of $1.6 \times 10^{7} / \mathrm{ml}$ with $12.5 \%$ CD95 ligand-containing supernatant. Apoptosis in the culture was monitored at regular intervals by staining aliquots of the cells with Hoechst H-33342 $(500 \mathrm{ng} / \mathrm{ml})$ and scoring cells with condensed or fragmented nuclei under the fluorescence microscope. After $90 \mathrm{~min}$, the cells were harvested by centrifugation at $4{ }^{\circ} \mathrm{C}$, and carefully washed twice with cold $\mathrm{PBS}^{2}$ and once with cold CEB buffer (50 mM Hepes $\mathrm{NaOH}$, pH 7.4, $50 \mathrm{mM}$ $\mathrm{KCl}, 5 \mathrm{mM}$ EGTA, $2 \mathrm{mM} \mathrm{MgCl}_{2}$ ) supplemented with $1 \mathrm{mM}$ dithiothreitol and protease inhibitors $(1 \mathrm{mM}$ phenylmethylsulfonyl fluoride, $100 \mu \mathrm{g} / \mathrm{ml}$ each of leupeptin, pepstatin, and aprotinin). The cells were then resuspended in a volume of CEB buffer equal to the packed cell pellet (for Jurkat cells this corresponds approximately to $100 \mu 1$ per $1 \times 10^{8}$ cells). The cell suspension was immediately frozen in liquid nitrogen, thawed in a water bath at $37^{\circ} \mathrm{C}$, and centrifuged for $10 \mathrm{~min}$ at $4{ }^{\circ} \mathrm{C}$ and $20,000 \mathrm{~g}$. The centrifugation step was repeated to obtain a clear supernatant, which was then snap-frozen and stored at $-80^{\circ} \mathrm{C}$. Cytosolic extracts from untreated cells were prepared using the identical procedure.

\section{Permeability assay}

HeLa 229 cells were seeded in 12-well plates containing glass coverslips at a density of $1.5 \times 10^{5} / \mathrm{ml}$. After $1216 \mathrm{~h}$ incubation (i.e., $5060 \%$ confluency) the coverslips were transferred to a 6-well plate on ice, washed with $5 \mathrm{ml}$ cold PBS, and then incubated in $5 \mathrm{ml}$ permeabilization buffer $(20 \mathrm{mM}$ Hepes $\mathrm{NaOH}, \mathrm{pH} 7.5$, $110 \mathrm{mM}$ potassium acetate, $5 \mathrm{mM}$ magnesium diacetate, $0.5 \mathrm{mM}$ EGTA, $250 \mathrm{mM}$ sucrose) containing $40 \mu \mathrm{g} / \mathrm{ml}$ digitonin for $45 \mathrm{~min}$. After removal of the digitonin solution the coverslips were washed three times with cold permeabilization buffer for 1, 5, and $10 \mathrm{~min}$ and briefly equilibrated in transport buffer $(20 \mathrm{mM}$ Hepes $\mathrm{NaOH}$, pH $7.3,110 \mathrm{mM}$ potassium acetate, $5 \mathrm{mM}$ sodium acetate, $2 \mathrm{mM}$ magnesium diacetate, $0.5 \mathrm{mM}$ EGTA, $250 \mathrm{mM}$ sucrose). The coverslips were then

\footnotetext{
${ }^{2}$ Abbreviations used: PBS, phosphate buffered saline; NPA, nu clear permeability assay; FRAP, fluorescence recovery after photo bleaching.
} 
placed in a humidified chamber and incubated for 5 7 min with $30 \mu \mathrm{l}$ of a mix containing cytosolic extract (corresponding to $300400 \mu \mathrm{g}$ of total protein) plus $1.5 \mu \mathrm{l}$ of an energy-regenerating system ( $1 \mathrm{mM}$ Hepes $\mathrm{NaOH}, \mathrm{pH} 7.3,0.5 \mathrm{mM}$ ATP, $0.5 \mathrm{mM}$ GTP, $12.5 \mathrm{mM}$ glucose, $10 \mathrm{mM}$ phosphocreatine, $0.3 \mathrm{U} / \mathrm{ml}$ creatine kinase) in transport buffer. Then $10 \mu \mathrm{l}$ of a $0.6-\mathrm{mg} / \mathrm{ml}$ dextran solution in transport buffer was added and homogeneously distributed. After another $57 \mathrm{~min}$ the coverslips were mounted in a coverslip holder and imaged by confocal microscopy.

Images were taken using a Leica TCS confocal microscope with a $40 \times$ oil immersion objective at constant pinhole and laser power settings. The size of the scanned area was $250 \times 250 \mu \mathrm{m}$. Eight randomly distributed, nonoverlapping fields were scanned per coverslip during a maximum time period of $5 \mathrm{~min}$.

\section{Image analysis and statistics}

Images obtained with the confocal microscope $(512 \times 512$ pixel $)$ were analyzed using the Nuclear Permeability Assay (NPA) ${ }^{3}$ software. An improvement procedure was performed to define the software settings using 30 sample images. The same parameter values were employed for the evaluation of images from different experiments. For each experiment 8 images were recorded. Experiments were done in triplicate. Statistical analysis was performed using Microsoft Excel.

\section{Results}

\section{Principle of the in vitro assay}

Exposure of HeLa cells grown on coverslips to low concentrations of digitonin results in the perforation of the plasma membrane but leaves the nuclear membrane intact (Fig. 1). For the detection of nuclear envelope permeability changes in apoptosis, the semipermeabilized cells are incubated with cytosolic extracts from either untreated or apoptotically stimulated Jurkat donor cells in the presence of $70-\mathrm{kDa}$ fluorescent dextran. Dextrans are spherical, hydrophilic molecules which are commonly used as probes for diffusion, since their binding to cellular structures is negligible compared to proteins of the same molecular size [26]. After a short incubation, the cells are imaged with the confocal microscope. Since the fluorescent reporter can freely diffuse through the plasma membrane, the cytoplasmic compartment shows a high fluorescence intensity and is barely recognizable against the background. Conversely, the intact nuclear membrane restricts the diffusion of the

\footnotetext{
${ }^{3}$ The NPA software is available as free download at http:// gutenberg.biologie.uni konstanz.de.
}

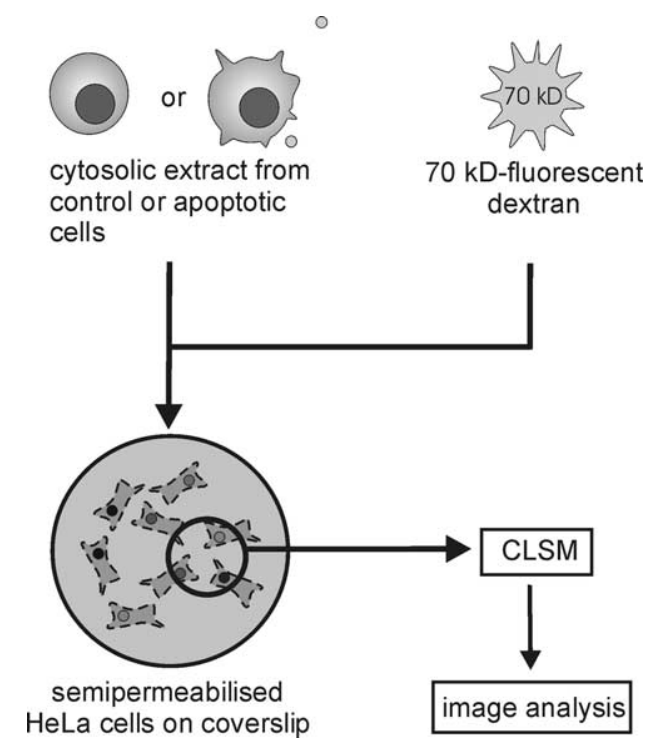

Fig. 1. Schematic of the nuclear permeability assay. Cytosolic extract isolated from either control or apoptotic cells is applied to a coverslip carrying semipermeabilized HeLa cells in presence of a fluorescent $70 \mathrm{kDa}$ dextran. Images are recorded with a confocal laser scanning microscope (CLSM) and analyzed by a dedicated software.

fluorescent dextran into the nucleus. The nuclei thus appear as dark circles. The degree of exclusion depends on the sieving properties of the nuclear membrane, which are mainly determined by the opening diameter of the nuclear pore. Incubation with cytosol of apoptotic origin increases the permeability compared to incubation with control cytosol. This is reflected by an increment of the percentage of bright nuclei in the microscopic image.

\section{Apoptotic model}

Jurkat cells were chosen as a source of cytosolic extracts, since they can be induced to undergo apoptosis by engagement of the death receptor CD95. This leads to a rapid and robust apoptotic response, whose underlying signal pathway has been elucidated in great detail [27,28]. Fig. 2 shows the time course of cell death in Jurkat cells treated with CD95 ligand. In the experiments reported here, extracts were harvested $90 \mathrm{~min}$ after stimulation, i.e., at a time when cell death in the population was below $20 \%$.

\section{Image recording and characteristics}

Images of the nuclear permeability assay were recorded using a laser scan confocal microscope to detect the amount of dextran present in a thin layer corresponding to the cross section of the nuclei. A maximum of eight nonoverlapping, randomly distributed fields were imaged per coverslip during a time period of no longer than $5 \mathrm{~min}$, to minimize artifacts due to photodestruction. 


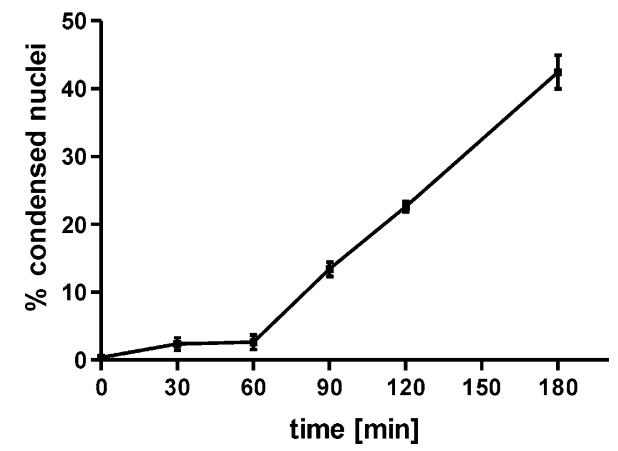

Fig. 2. Time course of the apoptotic response of Jurkat cells treated with CD95 ligand. At the time points indicated, aliquots of the culture were stained with Hoechst $\mathrm{H} 33342$ and scored under the fluorescence microscope for the presence of condensed nuclei.

Fig. 3 shows typical images of the permeability assay performed either under control conditions (Fig. 3a) or with apoptotic extract (Fig. 3d). Closer inspection of these images reveals the presence of at least three different type of objects: (1) "ideal" objects, round or slightly elliptical nuclei with a sharp margin and a lower level of fluorescence compared to the background (Figs. $3 \mathrm{~b}$ and e), (2) sharp-angled objects which represent cells whose plasma membrane has not been permeabilized by

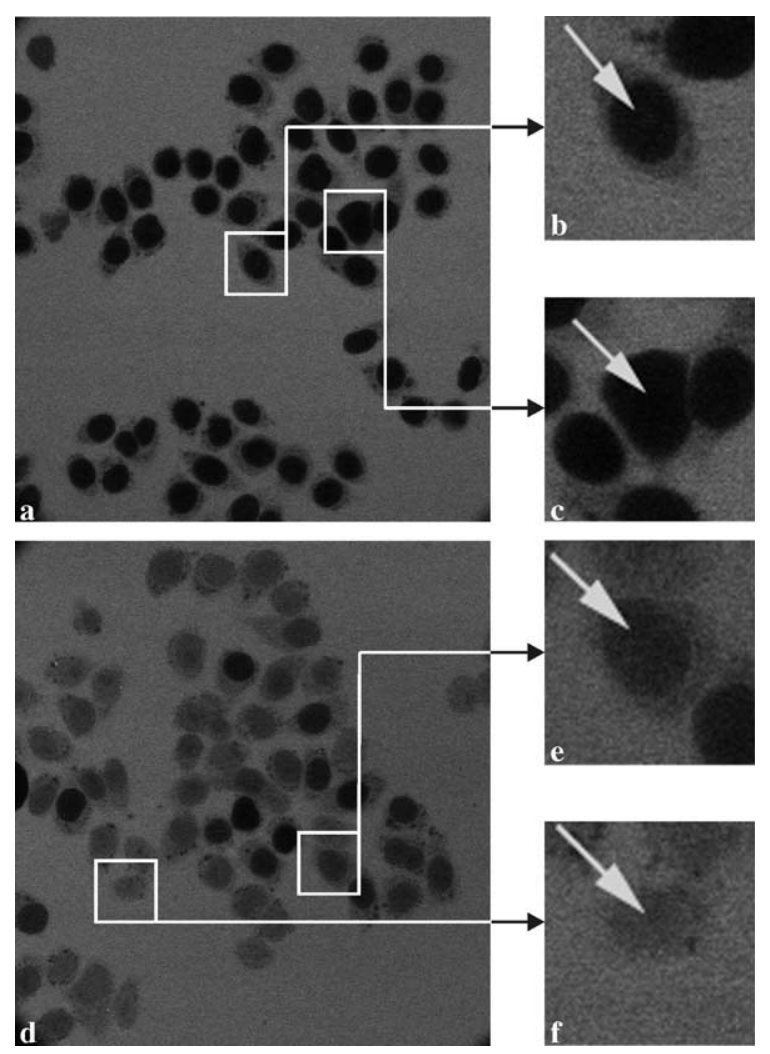

Fig. 3. Confocal images of the nuclear permeability assay. (a) Incu bation with control cytosolic extract. (d) Incubation with apoptotic extract. (b f) Object classification: (b) and (e) "ideal" objects, nuclei in the focal plane; (c) nonpermeabilized cell; (f) out of focus nucleus. digitonin and thus exclude the fluorescent marker from the cytoplasm (Fig. 3c), and (3) objects with blurred margins which represent nuclei outside of the focal plane of the image (Fig. 3f). Class 2 and 3 objects were always present in low numbers due to the individual variability of the cells with regard to shape, adherence, and susceptibility to digitonin treatment.

\section{NPA software}

The aim of the assay was the quantitative comparison of the nuclear fluorescence signal under control and apoptotic conditions. Since this is best achieved by computer-assisted analysis, we undertook the effort to develop a suitable dedicated software. An algorithm which recognizes all potentially interesting objects (i.e., nuclei of permeabilized cells) and then selects them for calculation of the corrected mean fluorescence signal was designed. The selection is based on the abovementioned criteria for the definition of different object classes. The recognition algorithm proceeds in several steps. First, it finds a threshold gray value for the areas corresponding to the cytoplasm and defines the remaining area as background. Inside the cytoplasm the algorithm then searches for darker regions (lower gray values) and accepts these as nuclei if their size is above a minimal threshold. The identified nuclei are then scored for the relation between their size and their form (round vs angled) and for the presence of surrounding cytoplasm (N.B. Out-of-focus objects have no or only a small cytoplasmic rim). Finally, the software calculates the mean grayscale value of the accepted nuclei $[\mathrm{x}]$ and divides it by the mean grayscale value of the background $[\mathrm{BG}]$. The results are saved automatically in a corresponding text file (results.txt) and can be imported in any common data analysis program.

By varying the threshold gray values for the recognition of cytoplasm ("Plasma") and nuclei ("Nucleus") and the value defining the roundness of an object ("Form"), the algorithm can be adapted to the characteristics of the image to be analyzed. Increasing values for "Plasma" and "Nucleus" will cause lighter areas to be identified as either cytoplasm or nuclei. Changing the "Form" parameter to values different from 1 allows for the recognition of shapes deviating from a perfect circle.

Fig. 4 shows screenshots of the software NPA. When started, the program opens a window with several buttons. The analysis is started by clicking on the "Open" button and choosing an image from the dialog box. The software automatically analyzes the selected image and displays it in two versions: In the right panel the recognized objects are marked by colored outlines. This is also an interactive window, where the object selection can be altered manually. On the left only those objects which are included in the calculation of the fluorescence signal are indicated. They are labeled by a blue outline. 
A click on the "Results" button below the left panel opens a box showing the intensity histogram of the image, the number of correctly recognized nuclei, the mean grayscale values of the nucleoplasm and the background, and the ratio x/BG ("Quotient"). On the right, the "Legend" button displays the color coding of the selection (see below), while the button "Original Image" opens an additional window containing the unmodified image. Activating the Settings button enables one to modify the parameters of the analysis, as mentioned above, by moving the corresponding slide controls. Before opening the "Settings" box, the user is reminded that this may change the outcome of the analysis. A new analysis with modified settings can be started by clicking on the "Analyze" button, the process can be interrupted by "Cancel."

The image displayed in Fig. 4 originates from a coverslip incubated with control cytosol. In this sample most nuclei are similar in size and shape, have a uniformly dark nucleoplasm, and are in the same focal plane. The recognition rate is high, resulting in almost all nuclei being circumscribed by a blue line. All of them are "ideal" objects included in the calculation of the mean grayscale value of the nucleoplasm.

In Fig. 5 the NPA software was applied to a "problematic" image, which contains cells which have not been fully permeabilized by digitonin, nuclei with different intensities, and out-of-focus objects. This is reflected by the high ratio of colored vs blue-rimmed nuclei in the right panel. Only the latter are considered for the calculation of the mean grayscale value. The remainder are excluded because they do not fit one or more of the following four criteria indicated in the legend box:

(i) size: the object is too large (purple);

(ii) border: the object is at the edge and not entirely included in the image (green);

(iii) plasma: the cytoplasmic rim around the object is below the threshold (red); and

(iv) form: the object has an irregular shape (yellow).

The selection can be altered manually by clicking on the desired nucleus in the interactive right window and choosing the corresponding option in the dialog box "Change Status of Nucleus" (Fig. 4). Manually excluded nuclei are then labeled by a dashed white outline and manually included nuclei by a dashed blue outline. The calculation is automatically adjusted to the new selection.

The software thus features two different tools to optimize the recognition of a given image. First, the threshold grayscale values of the different areas plasma and nucleus and the form parameter can be varied. This will influence both the recognition and the selection of the nuclei. Second, subsequent to the analysis the manual tool can be used to correct the selection within a given set of recognized objects. Whenever these tools are employed, the results must be verified by statistical analysis (see below).

The image shown in Fig. 5a has been analyzed with default settings. Fig. 5b shows the result of a new analysis using improved values for the parameters "Plasma," "Nucleus," and "Form" and the subsequent employment of the manual selection tool. How this affects the recognition of individual critical objects is shown in detail in Fig. 5c. In the improved evaluation the number of correctly recognized objects increases from 21 to 33 .

\section{Improvement of the software settings}

A prerequisite for the application of the NPA software is the determination and validation of a consensus set of values for the parameters "Plasma," "Nucleus," and "Form," which will allow for the correct analysis of most if not all images obtained within a given experimental setup. It is crucial to use the same settings for all images to be analyzed to minimize bias and avoid a potentially significant alteration of the results.

The protocol used for the determination of optimal settings is depicted in Fig. 6. Thirty images taken from coverslips incubated with control cytosol were first analyzed with default values (Nucleus $=1$, Plasma $=1$, Form $=1$ ) using the manual tool to adjust the selection. For the same images, the slide controls in the setting box were then used to determine the settings which resulted in an "optimal" recognition of each individual image, yielding the maximum number of recognized nuclei while still excluding "false" objects. If necessary, the manual tool was employed to correct the selection. For reasons of comparison, it was important to obtain approximately the same number of selected nuclei as that in the analysis which used default parameters plus the manual selection tool. The results derived from the analyses with default settings and with improved settings were then compared in a two-tailed paired $t$ test. As shown in Fig. 6, the difference between the two data sets was statistically significant. This indicated on the one hand that the default values do not suit the analysis of the sample images. On the other hand it also highlights the sensitivity of the software toward variation of the settings values.

To define a new set of parameters, the 30 improved values of each parameter were averaged and the mean values were used to reanalyze the sample images. Comparison of these results with those from the nonaveraged analysis in a two-tailed paired $t$ test yielded a difference below statistical significance. The mean values were thus defined as new defaults for the analysis of the permeabilization assay and written to the configuration file.

To ensure that these settings would also properly recognize images obtained from coverslips incubated with 

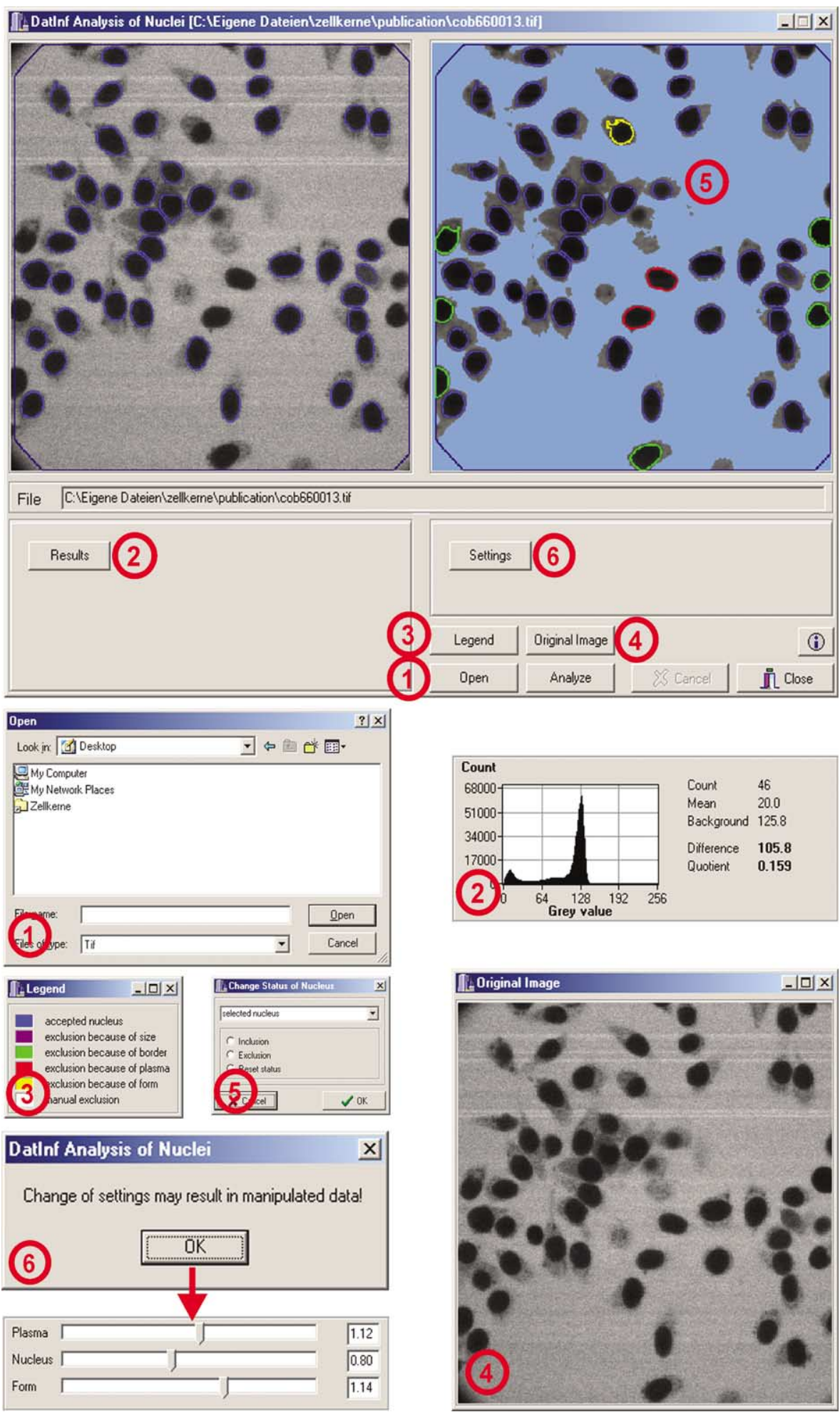

Fig. 4. Layout of the NPA software. The main window is shown on top. In the right panel all nuclei that are recognized are encircled. In the left panel only those nuclei, that are used for the calculation of the mean grayscale value of the nucleoplasm are indicated in blue. The buttons open different boxes, which are indicated by the corresponding numbers.

apoptotic cytosol, the optimal settings for 30 "apoptotic" images were individually determined as described above for the control images. The results obtained from this analysis and from the analysis performed with the new default values were compared in a two-tailed paired $t$ test. The statistical analysis did not yield any significant 

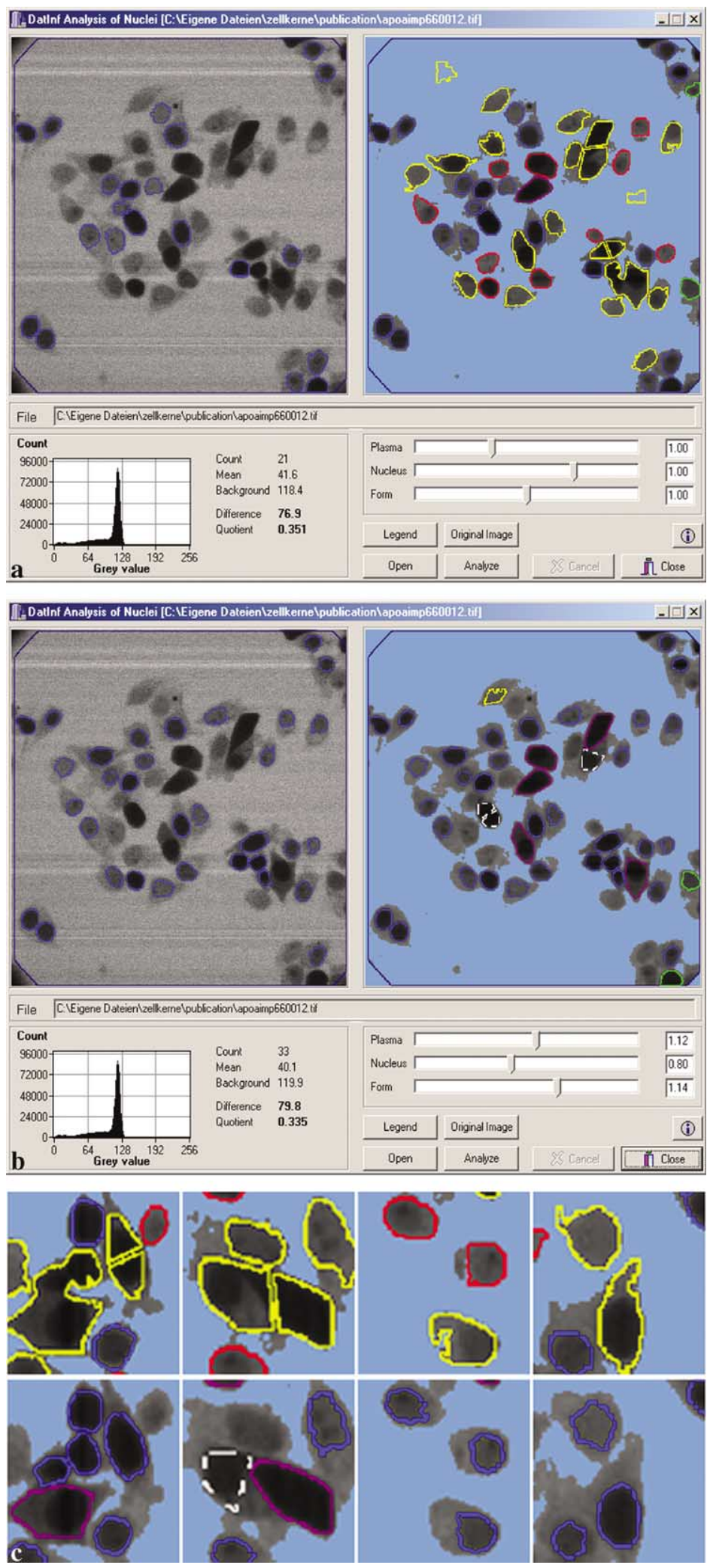

Fig. 5. Image analysis by the NPA software. (a) Analysis with default settings (b) Analysis with improved settings (c) Selective enlargements from (a) and (b); comparison of the object recognition with default settings (top row) and improved settings (bottom row). The colored rims indicate the status of the nuclei. Blue rimmed nuclei are automatically included in the calculation of the mean fluorescence intensity of the nucleoplasm. Nuclei encircled by a dashed white outline have been included with the manual selection tool. The remainder are excluded because of the following reasons: yellow, object excluded because of irregular shape; red, object excluded because it is not surrounded by cytoplasm; purple, object excluded because it is too large. 

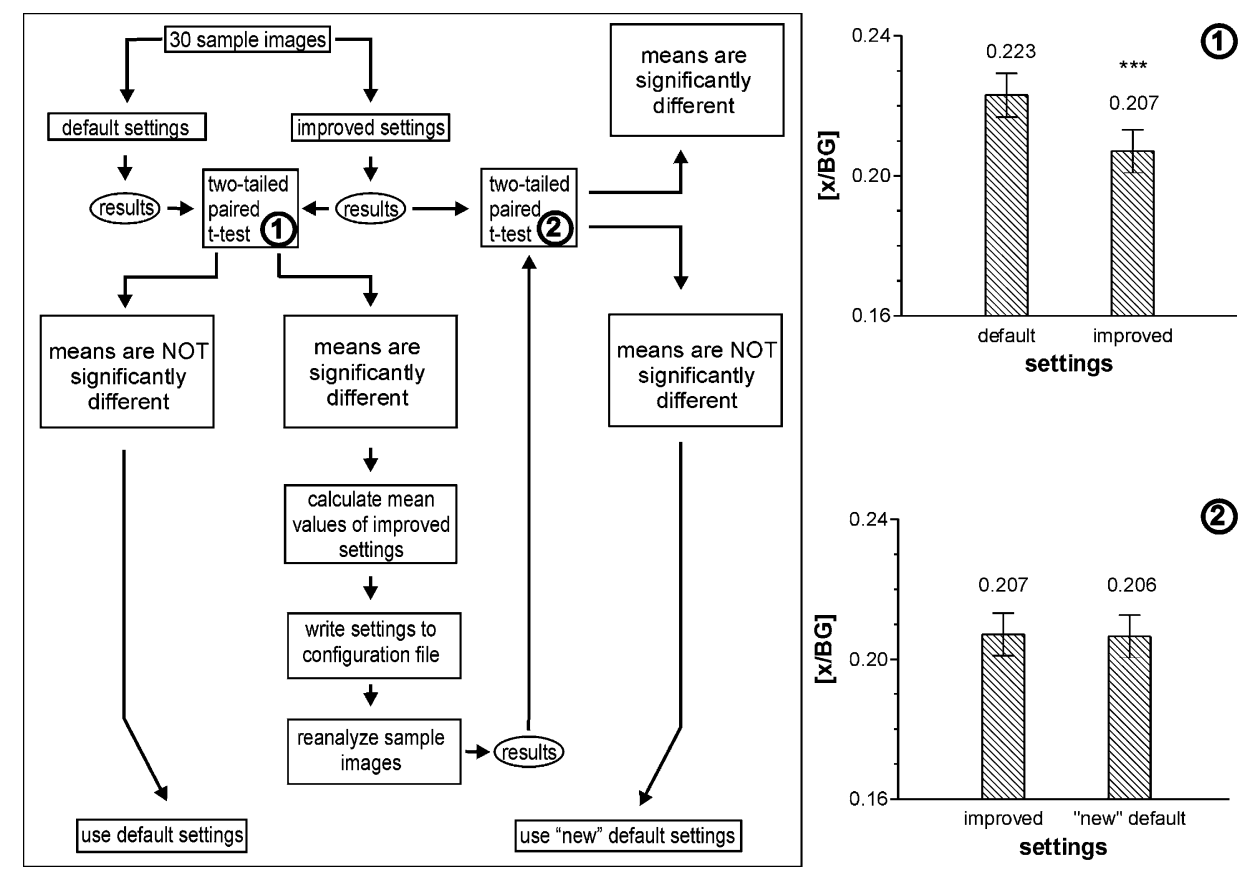

Fig. 6. Protocol for parameter adjustment. The bar diagrams show the results of the two tailed $t$ tests indicated in the flowchart. [x/BG], corrected fluorescence intensity of the nucleoplasm, ${ }^{* * *} p<0.0001$.

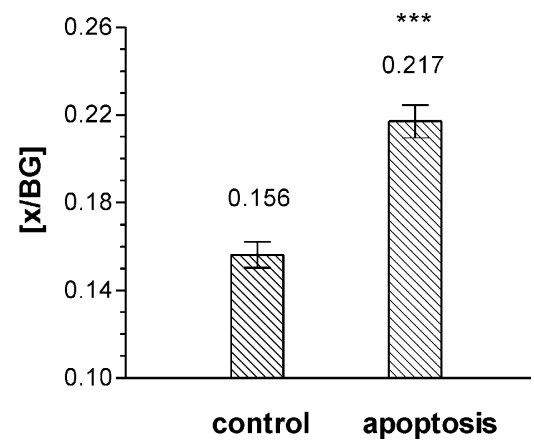

Fig. 7. Comparison of the fluorescence intensity of the nucleoplasm in nuclei exposed to cytosolic extracts from untreated and apoptotically stimulated cells. Each data set represents the average of 10 images. The experiment was performed in triplicate with similar results. $[\mathrm{x} / \mathrm{BG}]$, corrected fluorescence intensity of the nucleoplasm. The results were analyzed with a one tailed $t$ test; ${ }^{* * *} p<0.0001$.

difference between the two sets of data, indicating that the identified set of parameters can be generally employed to evaluate the nuclear permeability assay.

\section{Passive nuclear permeability is altered under apoptotic conditions}

Having established the conditions for the analysis of the images, we set out to measure whether incubation of the nuclei with cytosol extracted from an apoptotic culture would alter the permeability of the nuclear envelope to $70-\mathrm{kDa}$ dextran compared to incubation with a control extract. For this experiment we performed a new set of assays. As shown in Fig. 7, analysis of the corresponding images yielded a significant increase in the fluorescence of the nucleoplasm. This result is in agreement with our previous observations in intact cells, where we could detect an increase in the permeability of the nuclear envelope to microinjected $70-\mathrm{kDa}$ dextran shortly after apoptotic stimulation [22].

\section{Discussion}

Molecular passage across the nuclear membrane has been the subject of scientific interest for almost 40 years [29]. Nevertheless, only few methods to investigate this essential cellular process are available and most of them are quite demanding in terms of equipment and expertise. Early studies were performed in amphibian oocytes, which were amenable to microinjection and could easily be enucleated to determine the nucleus-to-cytoplasm distribution of the microinjected molecule. These were usually radiolabeled proteins or dextrans. Their partition coefficient was determined by autoradiography of oocyte sections $[26,30]$ or by gamma counting of the separated nucleus and cytoplasm [31]. Alternatively, gold-labeled particles or proteins were injected and their distribution was detected by electron microscopy $[32,33]$.

Studies on nonfixed specimens became possible with the advent of specialized fluorescence microscopy techniques, in particular fluorescence recovery after photobleaching (FRAP). First applied to the study of nuclear 
envelope permeability by Peters [34], FRAP has been used to measure diffusion into isolated nuclei [35] and into the nuclei of intact cells $[4,36]$. A recent development of this technique is optical single transporter recording, which allows one to measure the permeability of single nuclear pores in isolated nuclear membrane patches [37].

To circumvent the requirement for microinjection when investigating nucleocytoplasmic trafficking in situ, two main strategies have been pursued. The first relies on the expression of fluorescent transport reporters and was originally developed for yeast cells [38] and later modified for eukaryotic cells [39]. The second and probably most widely used method is the in vitro import assay established by Adam et al. [24]. In contrast to the methods described above, which yielded insights into the biophysical properties of the nuclear pore and the kinetics of the translocation process, this assay has been used as a biochemical endpoint for the molecular characterization of the machinery responsible for signalmediated transport. Several key nuclear transport factors and the energy requirements of nuclear import have been elucidated using this approach. Still, a major limitation of the semipermeabilized cell assay is the lack of quantitative readout. Usually, microscopic images of the assay showing nuclei of semipermeabilized cells are compared qualitatively. This is feasible whenever clearcut effects are observed. However, subtle variations in nuclear fluorescence are difficult to grasp. The solution in this case is to analyze the signal with an image analysis program. Such programs usually require the manual definition of regions of interests corresponding to individual nuclei, which is a time-consuming and tedious procedure.

In this work, we have sought to apply the semipermeabilized cell assay to the analysis of passive diffusion processes, which yield only subtle changes in nuclear fluorescence intensity. To this end we have developed an image analysis software that is specifically suited to the recognition of nuclei in semipermeabilized cells grown on coverslips. Since the images may vary with respect to the overall fluorescence, the ratio of nuclear-to-cytoplasmic signal, and the shape and density of the cells, we have introduced some degree of flexibility, which allows the user to set a configuration that matches the experimental requirements. The major adaptation step consists in finding the threshold values for the nuclear and cytoplasmic fluorescence and the value defining the degree of roundness of the objects to be recognized. We have also added the possibility to manually exclude or include nuclei. This tool has proven essential for the parameter adjustment procedure, since it allows one to compare evaluations performed with different parameter settings while keeping the number of analyzed objects constant. However, once the optimal set of values has been determined, use of the manual tool can be minimized or omitted altogether. We strongly recommend performance of the adjustment protocol with a sufficient number of images and validation of the results by statistical analysis as described in the flowchart in Fig. 6. Once this has been performed, the images can be automatically evaluated, allowing for the quantitation of a large series of experiments in a short period of time.

In a trial application we were able to detect a significant increase in passive nuclear permeability induced by cytosolic extracts of cells committed to undergo apoptosis. These extracts were isolated in the early phase of the apoptotic response, when less then $20 \%$ of the cell population had undergone cell death, to avoid disruption of the nuclear pore by caspases [22]. In fact, when the semipermeabilized cells are incubated with extracts from late apoptotic time points they immediately detach from the coverslip, impeding microscopic analysis. Interestingly, the increase in nuclear permeability observed here appears to be caspase independent, since it could not be inhibited by a pan-caspase inhibitor (A. Tabbert et al., unpublished).

The mechanisms underlying the apoptotic modulation of pore permeability are still unknown. Since small alterations of the pore diameter are predicted to produce large changes in the rate of protein entry [26] it is likely that the effects observed will have a significant impact on the signaling of the cell death process. In fact, a number of relevant proapoptotic factors have been recently described to move in and out of the nucleus either preceeding or independent of the activation of caspases [40 43]. The assay described here represents a powerful tool for the biochemical isolation of the factors responsible for the modulation of nuclear permeability from apoptotic cytosolic extracts. Through its flexibility it will also be useful in other applications, focusing either on passive nuclear permeability or on signalmediated active transport.

\section{Acknowledgments}

We gratefully acknowledge the support of Pierluigi Nicotera, in whose laboratory this work was initiated, and thank Irene Biller-Ckovric for technical assistance, Dirk Görlich for help with the semipermeabilized cell assay, and Alexander Bürkle for critical reading of the manuscript. We also thank Holger Lüdtke and Ulf Ellwanger (DatInf $\mathrm{GmbH}$ ) for the fruitful teamwork on the software development.

\section{References}

[1] I.G. Macara, Transport into and out of the nucleus, Microbiol. Mol. Biol. Rev. 65 (2001) 570594. 
[2] D. Gorlich, U. Kutay, Transport between the cell nucleus and the cytoplasm, Annu. Rev. Cell Dev. Biol. 15 (1999) 607660.

[3] E. Kiseleva, M.W. Goldberg, J. Cronshaw, T.D. Allen, The nuclear pore complex: structure, function, and dynamics, Crit. Rev. Eukaryot. Gene Expr. 10 (2000) 101112.

[4] L.W. Jiang, M. Schindler, Nuclear transport in 3T3 fibroblasts: effects of growth factors, transformation, and cell shape, J. Cell Biol. 106 (1988) 1319.

[5] C.M. Feldherr, D. Akin, The permeability of the nuclear envelope in dividing and nondividing cell cultures, J. Cell Biol. 111 (1990) 18.

[6] C.M. Feldherr, D. Akin, Signal mediated nuclear transport in proliferating and growth arrested BALB/c 3 T3 cells, J. Cell Biol. 115 (1991) 933939.

[7] C.M. Feldherr, R.E. Lanford, D. Akin, Signal mediated nuclear transport in simian virus 40 transformed cells is regulated by large tumor antigen, Proc. Natl. Acad. Sci. USA 89 (1992) 11002 11005 .

[8] E. Kiseleva, M.W. Goldberg, B. Daneholt, T.D. Allen, RNP export is mediated by structural reorganization of the nuclear pore basket, J. Mol. Biol. 260 (1996) 304311.

[9] B. Daneholt, A look at messenger RNP moving through the nuclear pore, Cell 88 (1997) 585588.

[10] T. Danker, H. Oberleithner, Nuclear pore function viewed with atomic force microscopy, Pflugers Arch. 439 (2000) 671681.

[11] H. Wang, D.E. Clapham, Conformational changes of the in situ nuclear pore complex, Biophys. J. 77 (1999) 241247.

[12] D. Stoffler, K.N. Goldie, B. Feja, U. Aebi, Calcium mediated structural changes of native nuclear pore complexes monitored by time lapse atomic force microscopy, J. Mol. Biol. 287 (1999) 741 752 .

[13] D. Moore Nichols, A. Arnott, R.C. Dunn, Regulation of nuclear pore complex conformation by IP(3) receptor activation, Biophys. J. 83 (2002) 14211428.

[14] L. Stehno Bittel, C. Perez Terzic, D.E. Clapham, Diffusion across the nuclear envelope inhibited by depletion of the nuclear $\mathrm{Ca}^{2+}$ store, Science 270 (1995) 18351838.

[15] M.A. Lee, R.C. Dunn, D.E. Clapham, L. Stehno Bittel, Calcium regulation of nuclear pore permeability, Cell Calcium 23 (1998) 91101.

[16] U.F. Greber, L. Gerace, Depletion of calcium from the lumen of endoplasmic reticulum reversibly inhibits passive diffusion and signal mediated transport into the nucleus, J. Cell Biol. 128 (1995) 514.

[17] C. Strubing, D.E. Clapham, Active nuclear import and export is independent of lumenal $\mathrm{Ca}^{2+}$ stores in intact mammalian cells, J. Gen. Physiol. 113 (1999) 239248.

[18] C.M. Feldherr, D. Akin, Regulation of nuclear transport in proliferating and quiescent cells, Exp. Cell Res. 205 (1993) 179 186.

[19] M.J. Clemens, M. Bushell, I.W. Jeffrey, V.M. Pain, S.J. Morley, Translation initiation factor modifications and the regulation of protein synthesis in apoptotic cells, Cell Death Differ. 7 (2000) 603615.

[20] M.L. Coleman, M.F. Olson, Rho GTPase signalling pathways in the morphological changes associated with apoptosis, Cell Death Differ. 9 (2002) 493504.

[21] P. Nicotera, S. Orrenius, The role of calcium in apoptosis, Cell Calcium 23 (1998) 173180.

[22] E. Ferrando May, V. Cordes, I. Biller, D. Gorlich, J. Mirkovic, P. Nicotera, Caspases mediate nucleoporin cleavage but not early redistribution of transport factors and mRNA in apoptosis, Cell Death Differ. 8 (2001) 495505.
[23] L. Faleiro, Y. Lazebnik, Caspases disrupt the nuclear cytoplasmic barrier, J. Cell Biol. 151 (2000) 951959.

[24] S.A. Adam, R.S. Marr, L. Gerace, Nuclear protein import in permeabilized mammalian cells requires soluble cytoplasmic factors, J. Cell Biol. 111 (1990) 807816.

[25] A. Rensing Ehl, K. Frei, R. Flury, B. Matiba, S.M. Mariani, M. Weller, P. Aebischer, P.H. Krammer, A. Fontana, Local Fas/ APO 1 (CD95) ligand mediated tumor cell killing in vivo, Eur. J. Immunol. 25 (1995) 22532258.

[26] P.L. Paine, L.C. Moore, S.B. Horowitz, Nuclear envelope permeability, Nature 254 (1975) 109114.

[27] P.H. Krammer, CD95's deadly mission in the immune system, Nature 407 (2000) 789795.

[28] H. Wajant, The Fas signaling pathway: more than a paradigm, Science 296 (2002) 16351636.

[29] C.M. Feldherr, Nucleocytoplasmic exchanges during cell division, J. Cell Biol. 31 (1966) 199203

[30] S.B. Horowitz, L.C. Moore, The nuclear permeability, intracel lular distribution, and diffusion of inulin in the amphibian oocyte, J. Cell Biol. 60 (1974) 405415

[31] W.M. Bonner, Protein migration into nuclei. I. Frog oocyte nuclei in vivo accumulate microinjected histones, allow entry to small proteins, and exclude large proteins, J. Cell Biol. 64 (1975) 421 430.

[32] C.M. Feldherr, A comparative study of nucleocytoplasmic inter actions, J. Cell Biol. 42 (1969) 841845

[33] C.M. Feldherr, The uptake of endogenous proteins by oocyte nuclei, Exp. Cell Res. 93 (1975) 411419.

[34] R. Peters, Nuclear envelope permeability measured by fluores cence microphotolysis of single liver cell nuclei, J. Biol. Chem. 258 (1983) 1142711429.

[35] M. Schindler, L.W. Jiang, Nuclear actin and myosin as control elements in nucleocytoplasmic transport, J. Cell Biol. 102 (1986) 859862.

[36] R. Peters, Nucleo cytoplasmic flux and intracellular mobility in single hepatocytes measured by fluorescence microphotolysis, EMBO J. 3 (1984) 18311836.

[37] O. Keminer, R. Peters, Permeability of single nuclear pores, Biophys. J. 77 (1999) 217228.

[38] N. Shulga, P. Roberts, Z. Gu, L. Spitz, M.M. Tabb, M. Nomura, D.S. Goldfarb, In vivo nuclear transport kinetics in Saccharomy ces cerevisiae: a role for heat shock protein 70 during targeting and translocation, J. Cell Biol. 135 (1996) 329339.

[39] S. Chatterjee, M. Javier, U. Stochaj, In vivo analysis of nuclear protein traffic in mammalian cells, Exp. Cell Res. 236 (1997) 346 350 .

[40] S.A. Susin, H.K. Lorenzo, N. Zamzami, I. Marzo, B.E. Snow, G.M. Brothers, J. Mangion, E. Jacotot, P. Costantini, M. Loeffler, N. Larochette, D.R. Goodlett, R. Aebersold, D.P. Siderovski, J.M. Penninger, G. Kroemer, Molecular characterization of mitochondrial apoptosis inducing factor, Nature 397 (1999) 441446.

[41] L.Y. Li, X. Luo, X. Wang, Endonuclease G is an apoptotic DNase when released from mitochondria, Nature 412 (2001) 95 99.

[42] N.D. Marchenko, A. Zaika, U.M. Moll, Death signal induced localization of $\mathrm{p} 53$ protein to mitochondria. A potential role in apoptotic signaling, J. Biol. Chem. 275 (2000) 1620216212.

[43] H. Li, S.K. Kolluri, J. Gu, M.I. Dawson, X. Cao, P.D. Hobbs, B. Lin, G. Chen, J. Lu, F. Lin, Z. Xie, J.A. Fontana, J.C. Reed, X. Zhang, Cytochrome $c$ release and apoptosis induced by mito chondrial targeting of nuclear orphan receptor TR3, Science 289 (2000) 11591164. 